Historic, archived document

Do not assume content reflects current scientific knowledge, policies, or practices. 



\title{
62.39
}

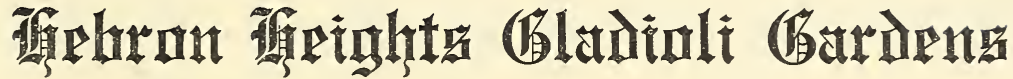

\author{
Frank Frenier, Hebronville, Mass: \\ MEMBER AMERICAN \\ MEMBER NEW ENGLAND \\ GLADIOLUS SOCIETY \\ GLADIOLUS SOCIETY
}

\section{Wholesale and Retail Price List for Fall and Winter 1924-1925}

\section{Please Read Carefully}

All stock is offered and orders accepted subject to prior sale or advance in price, in which event money will be refunded.

Reference: First National Bank of Attleboro, Mass.

I exercise the greatest care to have every variety true to name, and while I do not guarantee the crop, I will replace free of charge or refund price paid on any bulbs that prove untrue to name.

Terms: Cash or equivalent with order. If desired $25 \%$ with order, balance C. O. D. for Spring delivery.

All stocir is graded standard sizes. Six will be sold at dozen rate, 25 at 100 rate, 250 at 1,000 rate.

I do not substitute without permission, but give the grower a little leeway as to sizes, as I may be out of size ordered, but may be able to give good value of other sizes that will do just as well and save correspondence.

My stock is limited on some varieties and sizes, but if stock is wanted of sizes not listed, write me as I no doubt have a few Also I have small lots of many varieties not listed, some new choice Duner and Kundred varieties among them Correspondence solicited

Postage is paid on each and dozen lots, larger amounts at purchaser's expense.

Varieties marked with a $*$ can be furnished in 1,000 lots of sizes 1 and 2 and bulblets by the pecis and bushel 
America, soft lavender pink.

Alice Tiplady, prim., orange salmon....... 08

Baron J. Hulot, royal purple.

Crimson Glow, large brilliant crimson.....

Carman Silva, tall graceful wbite....... . .15

Evelyn Kirkland, rosy pink, a beauty....* .0S

Gretchen Zang, soft pinb .......... . 07

Halley, salmon pink............. . 05

Herada, lavender mauve. . . . . . . . . . .08

Lily White, early white ............. .06

Lucreata, earliest of all whites......... . .05

Le Mar Foch, color of America early....* .06

Louise, the queen of lavenders........ . .10

Mis. Francis King, scarlet.......... . . 05

Mrs. Frank Pendleton, salmon blotcb.... . 06

Mrs. Watt, American beauty color ...... . .06

Mrs. Dr. Norton, delicate cream pink.... . $\quad .10$

Miss Helen Franklin, $\pi$ bite ruffled . . . . . .06

Mary Pickford, white sulphur throat.... . . .10

Myra Prim, giant salmon........... . 10

Orange Glory, ruffled orange......... . .10

Pink Beauty, earliest of all glads....... . . 08

$\mathrm{P}_{1}$ ince of Wales, delicate salmon....... .08

Peace, large late white.............. .06

Panama, deep pink................ 05

Schwaben, suplhur yellow ............ . . . . .

The Pearl, pearly pink early .......... .05

Wilbrink, soft pink eərly.............. .08

Yellow Hammer, tall early yellow...... . . 08

1910 Rose, early rose, beautiful........ . 08

Mixed Varieties, all colors............ .05

Annie Eberius, velvety purple........ . .15

Autumn Queen, creamy fall tint..........

Albania, Kemp's fine wnite.............. .25

Bertrex, large early wnite........... .06

Byron L. Smith, laven der pink. . . . . . . . 10

Diana, early crimson extra.

Dr. W. Van Fleet, tall rose pink....... . .35

Europa, best cf all wbites............ .15

E. J. Shaylor, deep rose pink . ........ . . 10

Elora, $\pi$ bite with blotch in tbroat..... . . 25

Flora, large yellow ................ . 10

Frank J. Symnes, ruffled peach red. . . . . . . 10

Glendale, rich dark purple .............

Glory of Holland, white............ . . 05

Golden Measure, pure golden yellow..... .25 large

Golden Glory

Giant Nymph, large flower

Indian Maid, peach blossom nink
No. 1 No. 2 No. 3 No. 4

$\begin{array}{lllll}.50 & \$ 1.75 & \$ 1.50 & \$ 10.00 & \$ 8.00\end{array}$

.85

1.00

1. 50

4.25

4.00

3.25

22.50

4.50

3.50

10.00

4.00

27.50

3.25

3.00

8.00
2.75

25.00

45.00

.70

.50

.75

60

.50

.60

1.00

.50

.60

.60

1.00

.60

1. 00

.80

1. 00

.75

75

.60

.50

.50

50

.75

75

75

2.00

2.50

15.00

14.00

12.50

$3.25 \quad 2.75$

$2.75-2.25$

1.75

2.50

1.50
2.00

15.00

15.00

10.00

2.25

5.00

30.00

2.50

2.00

2.00

2.50

4.00

2.00

2.50

3.50

12.00

4.50

2.00

12.00

4.00

4.00

15.00

4.50

3.50

4.00

3.50

30.00

25.00

3.50

3.50

25.00

25.00

2. 50

3.00

17.50

2.00

1.75

17.50

12.00

2.25

2.00

12.00

2.50

2.00

10.00

3.00

2.50

15.00

3.00

16.00

17.50

$\begin{array}{llll}1.75 & 1.50 & 10.00 & 7.50 \\ \text { wing varieties are priced per } 100\end{array}$

$\begin{array}{llll}1.75 & 1.50 & 10.00 & 7.50 \\ \text { wing varieties are priced per } 100\end{array}$

18.00

20.00

20.00

35.00

10.00

8.00

8.00

10.00

8.00

7.50

7.50

22.50

6.00

7.50

8.00

15.00

10.00

20.00

16.00

16.00

12.00

10.00

10.00

8.00

8.00

8.00

12.00

10.00

12.00

The foll
1.50

.50

2.50

.60

1.00

1.00

1.50

1.50

1.00

2.50

1.00

1.00

2. 00

.40

1. 50

25

$\begin{array}{rrrr}1.75 & 1.50 & 5.00 & 4.00 \\ & & & 9.00 \\ 2.00 & 1.50 & 1.25 & 1.00 \\ 9.00 & 7.00 & 6.00 & 4.50 \\ 5.00 & 4.00 & 3.50 & 2.50 \\ 10.00 & 8.00 & 6.00 & 4.00 \\ & & & 12.00 \\ 8.00 & 6.00 & 3.50 & 3.00 \\ 6.00 & 4.50 & 3.50 & 3.00 \\ & & & 8.00 \\ 5.00 & 4.00 & 3.00 & 2.50\end{array}$

10.00

$1.50 \quad 1.25 \quad 1.00$

$10 \mathrm{~s}$
3.50

$10.00^{15}$ medium $7.50 \quad 5.00$

15.00

15.00

.

14.00

12.00

15.00

25.00

6.00

5.00

6.00

5.00

5.00

16.00

3.50

4.00

5.00

10.00

6.00

12.00

12.00

12.00

9.00

6.00

5.00

7.50

3.00

7.50

4.00

3.00

6.00

5.00

3.50

2.00

3.00

10.00

2.00

6.00

1.50

3.00

8.00

10.00
10.00

2. 50

2.50

3.00

5.00

4.00

7. 50

7.50

5.00

4.00

.00

4.00

2.50

2.00

8.00

1.50

5.00

1.00

2.00

5.00

.15

5

1.00

.40

1.00

.35

.15

.35

$.35 \quad 2.00$

1.00

.15

.50 
Each Dozen Per 100

Jach London, ligbt salmon......... .15

Kund's Mar. Foch, salmon pink. . . . . . . . .20

Kund's Glory, cream apricot......... .08

L. Immacalue, pure white.......... . .08

Mrs. O. W. Halliday, cream pink ...... . .10

Mrs. F. C. Peters, lilac rose, a wonder .... $\quad .30$

Mrs. W. E. Fryer, orange tint. .......... 08

Mrs. Wm. Kent, rose ash color......... . 10

Mis. H. E. Bothin, geranium pinl ...... . .15

Ma1y Fennell, deep lilac............ . 10

Master Wietze, violet.............. . .08

Maize, color of Wibrink Early .......... 08

Myrile, early pink.............. .08

Magic, lavender, purple throat........ . .15

Mona Lisa, soft rose pink .......... .15

Masterpiece, ruffled American beauty... $\quad .75$

Purple Glory, dark purple............... .50 large
Parliment, large pink, light throat.....

Parliment, large pink, light throat...... . . $10 \quad 1.00$

06

Pink Wonder, very large pink ......... . .20

Rosebud, lavender pink............ .08

Rose Ash, ashes of roses........... .25 large

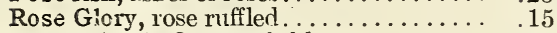

Rev. Eubank. finest soft blue......... . .15

Scarsdale, rosy lavender........... .06

Sentenial, large rose pink ............ .08

Sulphur Glow, intensely rufled. ....... .25

Smiles, light pink .............. 20

Sweet Lavender, light lavender....... . .20

Sheila, rich saimon .............. 20

Ticanic, lilac purple.............. . 15

Thos. T. Kent, strawberry pink....... .15

White Glory, rufiled white.......... .10

White Giant. shape or lily ............. .15

Write Wonder, large white.......... . 20

Youelis Favorite rusy pink .............

Arion, light salmon.

Butter Boy, butiereup sellow.......................

Concolor, tallest yellow.............. 08

Dorothy Wheeler, delicate pink........ . . 15

Golden Girl, colden yellow. . . . . . . . . . . 10

Maiciens Blush, blush pink........... . os

Roanoais, early vellow .............. 05

Sunbeam, early fine yellow .............. . 08

Wnite Butterf1, large white. . . . . . . . . $\quad .25$
1.50

2.00

.75

.75

.75

3.00

.75

1.00

1.50

1.00

.75

.75

.75

1.50

1.50

2.00

.75

arge

.60

.75

2.50

2.00

2.00

2.00

1.50

1.50

1.00

1.50

2.00

1.50

The following Premulinus varieties priced per 100

1.00

.75

1.50

1.00

.75

.50

2.50

$\begin{array}{llll}3.00 & 2.00 & 1.50 & 1.00\end{array}$

$\begin{array}{llll}3.00 & 2.50 & 2.00 & 1.50\end{array}$

5.00

3.00

35 medium

$5.00 \quad 4.00 \quad 3.00$

$\begin{array}{lll}3.00 & 2.50 & 2.00\end{array}$

6.00

$\begin{array}{llll}3.00 & 2.50 & 2.00 & 1.50\end{array}$

5.00

3.50

$6.00 \quad 5.00$

4.00

3.00

5.00

4.00

$\begin{array}{ll}4.00 & 3.00\end{array}$

3.00

2.50

2.50

2.00

2.50

1.75

1.00

$\begin{array}{rr}4.00 \quad 3.00 \quad & 2.00 \\ & 15.00\end{array}$

1.50

10.00
No. 1 No.

Nos. 3, 4, 5 and 6 per 100

Bulblets

No. 2 No. 3 No. 4 No. 5 No. 6 Per 100 Per 1000 Per Qt.

$\begin{array}{rrrrrrrr}3.00 & 2.50 & 2.00 & 1.50 & & & .15 & 1.00 \\ 5.00 & 4.00 & 3.00 & & & & & .35 \\ & & 15.00 & 10.00 & 7.00 & 5.00 & 2.00 & 15.00 \\ 3.00 & 2.50 & & & & & & \\ & & & & & & .35 & 2.00 \\ & & & & & & .75 & 6.00\end{array}$

$\begin{array}{lllll}4.00 & 3.50 & 3.00 & 2.00 & \\ 3.00 & 2.50 & 2.00 & 1.50 & .50\end{array}$

2.00
15.00

25 small bulb

1.50

1.1

1.00

$\begin{array}{lrr}4.00 & 3.00 \quad & 10.00\end{array}$

4.00

3.00

5.00

1.00

6.00

.10 small bulb

$7.00 \quad 6.00$
7.00

$7.00 \quad 5.00$

$\begin{array}{llll}2.50 & 2.00 & 1.50 & 1.00\end{array}$

8.00

6.00

6.00

6.00

2.50

2.00

2.50

4.00

.25

1.50

.50

2.50

.50

. .50

.75

.50

.50

5.00

4.00

4.00

$1.50 \quad 10.00$

1.50

1.00

$4.00 \quad 1.00$

1.50

.50

.50

1.00

1.50

3.00

.25

.25

.45
.40

.50

4.00

1.50

3.00

2.50

(5)

2.00

6.00

1.00

5.00

2.00

2.00
2.50

2.00

1.00

1.25

1.00

1.00

8.00

.75

6.00

1.50 


\section{Just a Few Cultural Notes}

The Gladiolus are so easy to grow that thay may be planted anywhere so long as they are in the sun, but they will not do well in any shade Don't try it.

Plant any time after the middle of April to the middle of June in any manner that best suits your plot of ground. Plant from 2 to 5 inches deep, the larger the bulb the deeper it should be planted. It stands up better

Now start the cultivator. The more the soil is kept stirred when not too wet, the better it conserves the moisture and your plants will do better.

The Gladioli will take a lot of water at blooming time. If you are in a position to water them at that time, do so. If not, they will do fairly well without, by keeping the soil well stirred up.

When cutting the flower keep as many leaves as possible on the plant to mature the bulb. Too low cutting weakens the bulb.

When digging, cut the stalk close to the bulb and dry well. When well dried and cleaned, store in cellar away from the furnace. If near to furnace, keep dish of water near them.

The flower of the Glaeioli is the finest for vases for the sicik rocm, hospitals, churches, cemetery and decorations of all kinds. Use them freely.

The flower growe1 is one of the best papers printed on Flower Growing. It is published monthly by Madison Cooper, Calcium, N. Y. Price $\$ 2.00$ per year

Become a member of the American Gladioli Society. It devotes its entire attention to the interests of the Gladioli and growers of the same over the entire country. Annual dues are $\$ 2.00$, payable to the secretary, John C. Davis, Rochester, N. Y. 